\title{
An Update on Some Structural Aspects of the Mighty Miniwall
}

\author{
ZDZISŁAW MARKIEWICZ* and MAGDALENA POPOWSKA \\ Department of Applied Microbiology, Institute of Microbiology, Poland \\ Received 15 July 2011, accepted 30 July 2011
}

\begin{abstract}
Peptidoglycan (PG), the mighty miniwall, is the main structural component of practically all bacterial cell envelopes and has been the subject of a wealth of research over the past 60 years, if only because its biosynthesis is the target of many antibiotics that have successfully been used in the treatment of bacterial infections. This review is mainly focused on the most recent achievements in research on the modification of PG glycan strands, which contribute to the resistance of bacteria to the host immune response to infection and to their own lytic enzymes, and on studies on the spatial organization of the macromolecule.
\end{abstract}

Ke y w ords: glycan strands, $N$-acetylglucosamine (GlcNAc) and $N$-acetylmuramic acid (MurNAc) modifications, peptidoglycan (PG), spatial organization

In 1884 Christian Gram devised a staining procedure that allowed classifying almost all bacteria to one of two groups, the Gram-positive and Gram-negative bacteria. The simple staining procedure is still widely used almost 130 years later, with practically all bacteria being classified to one group or the other. However, it was not until many years after Gram's invention that light was shed on the complexity of bacterial cell envelopes and the structures forming them. Studies of bacterial cell surfaces began in earnest in the 1950s and 1960s, following the isolation by Park and Johnston (1949) of what were later found to be cell wall PG precursors and the purification of bacterial cell walls by e.g. Salton $(1952,1957)$ and Work (1957). The term "microdermatology" was coined. It was found that all bacteria, except for some notable exceptions, i.e. mycoplasmas, Planctomyces and Orientia tsutsugamushi contain peptidoglycan (PG, syn. murein). PG has never been found in chlamydia either, although the bacteria have a functional pathway for meso-diaminopimelate, one of the unique structural building bricks of the macromolecule (Pavelka, 2007). More recently, an interesting new phylum of PG-free bacteria, the Verrucomicrobia, has been established (Yoon et al., 2010).

There has been a resurgence in studies on PG in the past few years fired, amongst others, by the increased prevalence of antibiotic resistance among bacteria that cause life-threatening infections and the need to find new agents that inhibit bacterial cell-wall biosynthesis (Bugg et al., 2011); the interaction of PG with innate immunity proteins (PG Recognition Proteins,
PGRPs or PGLYRPs) that are conserved from insects to animals and the mechanisms that lead to bacterial cell death (Dziarski and Gupta, 2010; Kietzman and Tuomanen, 2011); last but not least, the role of D-amino acids, which are a universal component of PG, in nature (Cava et al., 2011).

The main structure of PG involves linear glycan strands cross-linked by short peptides. "Normal", that is unaltered, glycan chains, have universally been found to be composed of alternating $\mathrm{N}$-acetylglucosamine (GlcNAc) and $N$-acetylmuramic acid (MurNAc) residues linked by $\beta-1 \rightarrow 4$ bonds. The D-lactoyl group of each MurNAc residue is substituted by a peptide stem whose composition is most often L-Ala- $\gamma$-D-Glu-mesoA2pm (or L-Lys)-D-Ala-D-Ala in nascent PG, the last $\mathrm{D}$-Ala residue being lost in the mature macromolecule. Cross-linking of the glycan strands generally occurs between the carboxyl group of $\mathrm{D}$-Ala at position 4 and the amino group of the diamino acid at position 3, either directly or through a short peptide bridge. The unique chemical traits of PG thus include the presence of $N$-acetylmuramic acid, $\gamma$-bonded D-Glu, L-D (and even D-D) bonds and non-protein amino acids, e.g. 2,6-diaminopimelic acid (A2pm) (e.g. Cummins, 1956; Work, 1957, 1961, 1969; Rogers, 1974; Glauner, 1988; Höltje and Glauner, 1990; Markiewicz et al., 1983; Markiewicz, 1993; Vollmer et al., 2011). These structural features are basically retained in all bacteria, though many differences in the glycan strands, composition of the stem peptide and/or interpeptide bridge are known and are taken into account in the tri-digital

\footnotetext{
* Corresponding author: Z. Markiewicz; e-mail: markiez@biol.uw.edu.pl
} 
PG classification system established by Schleifer and Kandler (1972). In some cases the differences may be quite extreme like, for example, the occurrence of mostly (75\%) A2pm $\rightarrow$ A2pm (i.e. $3 \rightarrow 3$ ) crosslinks in Clostridium difficile PG (Peltier et al., 2011)

Variations in the structure of the glycan strands of PG have recently been elegantly reviewed by Vollmer (2008). A review by Davis and Weiser (2011) focuses specifically on the role of peptidoglycan modifications and their effects on the host immune response to infection. A unique modification of glycan strands is the presence of muramic $\delta$-lactam, which occurs e.g. in the thick PG of Bacillus sp. and Clostridium sporogenes endospores. In Bacillus subtilis approximately every second MurNAc residue along the glycan strands is modified to muramic $\delta$-lactam. The modification of MurNAc involves the action of two enzymes, the MurNAc deacetylase PdaA and the amidase CwlD. Studies with mutants lacking these enzymes have shown that intact endospores are formed but that the spores are not able to germinate since unmodified spore PG is not recognized by germination-specific hydrolases (Popham et al., 1996, Atrih and Foster, 2001; Gilmore et al., 2004). More recently, it has been demonstrated that in Bacillus anthracis germination is mediated by the action of germination-specific lytic enzymes (GSLEs), one of which is SleB. SleB functions independently as a lytic transglycosylase on both intact and fragmented cortex. Most of the muropeptide products that SleB generates are large and are potential substrates for other GSLEs present in the spore, such as a glucosaminidase that cleaves between $\mathrm{N}$-acetylglucosamine and muramic- $\delta$-lactam. SleB has two domains, the $\mathrm{N}$-terminal domain is required for stable PG binding, while the C-terminal domain is the region of PG hydrolytic activity, which is dependent on cortex containing muramic- $\delta$-lactam in order to carry out hydrolysis (Dowd et al., 2008; Heffron et al., 2011).

PdaA, mentioned above, is an example of several $\mathrm{N}$-deacetylases found in different Gram-positive bacteria, which carry out the $\mathrm{N}$-deacetylation of MurNAc or GlcNAc (or both) in polymerized PG. N-deacetylation of MurNAc was found to protect bacterial cell walls from degradation by lysozyme, an important factor of the innate immune system (Araki et al., 1971). These enzymes, which have a predicted extracytoplasmic location in the cell, have been thoroughly reviewed by Vollmer (2008). The N-deacetylase of Streptococcus pneumoniae (PgdA) has been shown to be a putative virulence factor (Vollmer and Tomasz, 2002). Deacetylation of PG increases the positive charge of the cell wall, possibly contributing to protection of the pathogens against the binding of cationic antimicrobial peptides of the host organism. Similar observations were made more recently by Popowska et al. (2009) who found that a $p g d A$ mutant of Listeria monocytogenes was more prone to autolysis and was more susceptible to cationic antimicrobial peptides, and by Meyrand et al. (2007) for Lactococcus lactis. In other studies, a mutant strain of L. monocytogenes lacking PdaA activity induced a massive IFN-beta response in a TLR2 and Nod1-dependent manner and was rapidly destroyed within macrophage vacuoles (Boneca et al., 2007; Corr and O'Neill, 2009) and in bone-marrow derived macrophages, $p g d A$ mutants of L. monocytogenes demonstrated intracellular growth defects and increased induction of cytokine transcriptional responses that emanated from a phagosome and the cytosol (Rae et al., 2011). In Streptococcus suis expression of the pgdA gene was increased upon interaction of the bacterium with neutrophils in vitro as well as in vivo in experimentally inoculated mice, suggesting that $S$. suis may enhance PG N-deacetylation under these conditions. Evaluation of the $p g d A$ mutant in both the CD1 murine and the porcine models of infection revealed a significant contribution of the $p g d A$ gene to the virulence traits of S. suis (Fittipaldi et al., 2008). In an interesting study, it was found that neither PgdA inactivation nor PgdA overexpression in Lactobacillus lactis leading to different levels of PG deacetylation confers any advantage in the persistence of this bacterium in the gastrointestinal tract and its ability to enhance host immune responses (Watterlot et al., 2010). Bacterial $\mathrm{N}$-deacetylases have been considered to be exported enzymes but it has recently been reported that some of these enzymes may also be cytoplasmic, with a potential role in PG turnover and recycling (Popowska et al., 2011, for a very good review on turnover and recycling, see Reith and Mayer, 2011). A similar N-deacetylase lacking a signal peptide for secretion into the periplasmic space has been found in Helicobacter pylori (Shaik et al., 2011). The enzyme showed no in vitro activity on the typical polysaccharide substrates of peptidoglycan and results from crystallization and structure studies suggest that it binds a small molecule at the active site, even though the peptidoglycan of a HP0310 (syn. HpPgdA) knock-out mutant was characterized by higher degree of acetylation compared to the wild-type, along with increased susceptibility to lysozyme degradation.

$\mathrm{O}$-acetylation of MurNAc, similarly to N-deacetylation, is typically associated with bacterial resistance to lysozyme PG from degradation by lysozyme as well as by endogenous autolytic enzymes, e.g. the lytic transglycosylases. As a protective modification it is more ubiquitous than $N$-deacetylation and has been found to occur in many different bacterial species, both Grampositive and Gram-negative (Vollmer, 2008). An additional acetyl group is linked to the $\mathrm{C} 6-\mathrm{OH}$ of MurNAc to form a 2,6-N,O-diacetylo muramic acid residue. The ester bond of $O$-linked acetate is significantly weaker than the amide bond of $\mathrm{N}$-linked acetate. 
Two types of unrelated $O$-acetyltransferases have been described, corresponding to different mechanisms of peptidoglycan $\mathrm{O}$-acetylation (Clarke et al., 2000; Bera et al., 2005, Crisostomo et al., 2006). The first mechanism involves a single protein (an OatA-type enzyme) which performs both the transport of acetate across the membrane and its transfer onto the peptidoglycan. The second mechanism involves two proteins, one for acetate transport across the membrane and the other for catalyzing its transfer to MurNAc. The acetate transport genes of this system are unknown. There are several candidate genes for these $\mathrm{O}$-acetyltransferases (named Pat). A new peptidoglycan $O$-acetyltransferase has been found in E. coli. The enzyme, named PatB, $O$-acetylates peptidoglycan within the periplasm. This activity was found to be dependent upon a second protein, PatA, which functions to translocate acetate from the cytoplasm to the periplasm, demonstrating that the $\mathrm{O}$-acetylation of peptidoglycan in Neisseria gonorrhoeae, and other Gram-negative bacteria, requires a two component system (Moynihan and Clarke, 2010).

The $O$-acetyltransferase reaction is reversed by peptidoglycan $O$-acetyl esterase activity (Weadge et al., 2005; Vollmer, 2008). In Bacillus anthracis, in contrast to other bacteria, O-acetylation of peptidoglycan is combined with $\mathrm{N}$-deacetylation to confer resistance of cells to lysozyme and is conferred by two unrelated $\mathrm{O}$-acetyltransferases. Activity of the Pat $\mathrm{O}$-acetyl-transferases is also required for the separation of the daughter cells following bacterial division and for anchoring of one of the major S-layer proteins (Laaberki et al., 2011).

Until very recently it was thought that only the MurNAc residues in the $\mathrm{PG}$ polymer can be $\mathrm{O}$-acetylated. However, the presence of $\mathrm{O}$-acetylation on $\mathrm{N}$-acetylglucosamine (GlcNAc) in Lactobacillus plantarum PG has just been reported (Bernard et al., 2011). Detailed structural characterization of acetylated muropeptides released from L.plantarum PG revealed that both MurNAc and GlcNAc are $O$-acetylated in this species. These two PG modifications are carried out by two dedicated $O$-acetyltransferases, OatA and OatB, respectively. Analysis of the resistance of mutant strains to cell wall hydrolysis demonstrated that GlcNAc O-acetylation inhibits the activity of the major L.plantarum autolysin, $N$-acetylglucosaminidase Acm 2 . In this bacterial species, inactivation of oatA, encoding MurNAc $O$-acetyltransferase, resulted in marked sensitivity to lysozyme. Moreover, MurNAc over-O-acetylation was shown to activate autolysis through the putative $N$-acetylmuramoyl-l-alanine amidase LytH enzyme. In L. plantarum, two different $O$-acetyltransferases seem to play original and antagonistic roles in modulating the activity of endogenous autolysins.

Another kind of modification of muramic acid occurs in the PG of most genera of the order Actinomy- cetales that contain mycolic acids, e.g. Mycobacterium sp. In these bacteria muramic acid is $N$-glycolylated and not $N$-acetylated, and this modification is introduced during the synthesis of UDP-linked PG precursors, specifically into the last cytoplasmic precursor, UDP-MurNAc-pentapeptide. The $N$-glycolylated form arises through the action of an $N$-acetyl muramic acid hydroxylase (NamH) (Raymond et al., 2005) present only in the Actinomycetales. The importance of glycolylation has been elusive, with a hypothesis proposed based on studies with $M$. smegmatis nam $H$ mutants that it may protect PG from the action of lysozyme. However, recent findings (Coulombe et al., 2009) identify N-glycolyl MDP (Peptidoglycan-derived Muramyl Dipeptide) as more stimulatory than $N$-acetyl MDP at eliciting NOD2-mediated immune responses in the context of both an intact bacterium and as a pure compound, consistent with early observations attributing exceptional immunogenic activity to the mycobacterial cell wall. Disruption of namH in M. smegmatis nulled NOD2-mediated TNF secretion, which could be restored upon gene complementation. In mouse macrophages, $N$-glycolyl MDP was more potent than $N$-acetyl MDP at activating RIP2, nuclear factor kap$\mathrm{paB}$ and proinflammatory cytokine secretion. Finally, $N$-glycolyl MDP was found to be more efficacious than $N$-acetyl MDP at inducing ovalbumin-specific T cell immunity in a model of adjuvancy (Coulombe et al., 2009; Davis and Weiser, 2011).

A different modification of the glycan strands that can be found in the PG of many Gram-negative bacteria, but also in some Gram-positive ones is the presence of a 1,6-anhydroMurNAc residue at the end of the chain. These are formed by the action of lytic transglycosylases (LTs), that have the same bond specificity as lysozyme (Höltje et al., 1975). These ubiquitous enzymes are classified to one of four distinct families, based on sequence similarities and identified consensus motifs (Blackburn and Clarke, 2001). The importance of these enzymes is reflected in the fact that the bacterium Escherichia coli has six of them, representing different families and subfamilies. LTs can be viewed as space-making enzymes. They cleave glycosydic bonds within the PG sacculus to allow for a number of different processes to occur. They play an important a critical role in the expansion of the sacculus and consequent cell growth by creating sites for the insertion of PG precursors (Höltje, 1998). They are also required for PG turnover and recycling. In concert with amidases LTs function to split the septum, thereby permitting the separation of dividing cells (Heidrich et al., 2002). LTs have also been suggested to contribute to pathogenesis (reviewed in Cloud-Hansen et al., 2006). An important question has always been how large structures, e.g. protein complexes, penetrate the PG layer (Scheurwater 
and Clarke, 2008). LTs have always been implicated in these processes. This topic has been very well reviewed by Scheurwater and Burrows (2011).

Glycan strands of PG are also modified via the attachment of many different types of compounds and polymers to muramic acid, usually via a phosphodiester bond. The most notable of these are the teichoic and teichuronic acids as well as the arabinogalactans. Very rarely, structures are attached to GlcNAc, as in Streptococcus agalactiae. This topic is vast and well beyond the scope of this review.

Even though the structure of PG and the various species-specific and function-determined modifications of its structural elements have been thoroughly investigated, there are still numerous unresolved fundamental questions regarding the architecture of the peptidoglycan, i.e. the orientation of the glycan strands and stem peptides in relation to the surface and axes of a cell (Vollmer and Seligman, 2010). Various models for the spatial organization of peptidoglycan have been proposed over the years and currently two opposing models are considered: a model in which the glycan strands run parallel to the cytoplasmic membrane (the "classical" or "classical" model, e.g. Höltje, 1998; Pink et al., 2000) recently supported by experimental data by Gan etal. (2008) and Hayhurst et al. (2008) and the opposing "scaffold" model in which the glycan strands are oriented perpendicularly to the membrane (Dmitriev et al., 2003, 2004, 2005; Meroueh et al., 2006). Unraveling the architectural issues is compounded by the differences of the thickness of PG in Gramnegative versus Gram-positive cells, which is approximately 5-10 times thicker in the latter compared to the former and the fact that structure may be affected by i.e. growth conditions, gene activity (e.g. Höltje and Glauner, 1990; Vollmer et al., 2008; Korsak et al., 2005, 2010; Cava et al., 2011), antibiotic production or resistance (Sieradzki and Markiewicz, 2004; Schaberle et al., 2011) and many other factors. Moreover, recent studies using cryo-transmission electron microscopy (cryo-EM) have conclusively demonstrated the existence of the equivalent of the Gram-negative periplasmic space in at least B. subtilis and Staphylococcus aureus (Matias and Beveridge, 2005; 2006), which also complicates interpretation in spatial structure studies. The technique reveals in Gram-positive bacteria two different cell wall layers: an inner wall zone (IWZ) of low-electron density, whose main component is lipoteichoic acid (Matias and Beveridge, 2008), and a high-electron density outer wall zone (OWZ). In the "layered" model the glycan strands are believed to run parallel to the plasma membrane, arranged as hoops or helices around the short axis of the cell, resulting in a woven fabriclike structure (Verwer et al., 1978; Vollmer and Höltje, 2004; Vollmer et al., 2008). A recent study by Gan et al. (2008), in which frozen-hydrated sacculi from E. coli and Caulobacter crescentus were examined by electron cryotomography, confirmed the layered model, showing that in the Gram-negative PG sacculus a single layer of glycan strands lie parallel to the cell surface, roughly perpendicular to the long axis of the cell, encircling the cell in a disorganized hoop-like fashion. Their data also precluded the scaffold model. However, assuming that Gram-negative bacteria do have a single layer of PG, then how can one explain the difference in the thickness of E. coli PG versus Pseudomonas aeruginosa PG (approximately $2: 1$, respectively, for either dry or hydrated isolated sacculi (Vollmer and Seligman, 2010)? The organization of PG in ovococcoidal mutant Lactobacillus lactis cells lacking cell wall exopolysaccharides was studied using AFM (Atomic Force Microscopy) topographic and recognition imaging. Topographic images showed periodic ridges on the mutant surface that always ran parallel to the short cell axis. Recognition imaging demonstrated that these ridges consisted of peptidoglycan. The results are consistent with a PG organization in the plane perpendicular to the long axis of the cell (Andre et al., 2010). It would thus seem that the 3-D architecture of PG in both Gram-negative and Gram-positive cells is of the layered type. However, observations of isolated Bacillus subtilis PG using AFM show that, at least in this species, spatial organization is more complex (Hayhurst et al., 2008). This may be related to the existence of the IWZ in B. subtilis (Matias and Beveridge, 2005; Zuber et al., 2006) and the finding that the glycan strands of the bacterium are longer 50 times longer than previously calculated. The model of Hayhurst et al. (2008) proposes that during biosynthesis small numbers of glycan strands are polymerized and cross-linked to build a peptidoglycan "rope", which is coiled into a helix to form inner surface cable structures. The nascent helix (cable) is inserted into the cell wall by cross-links between two existing cables and the overlying cable interface cleaved by autolysins known to be essential for cell growth. As part of cable maturation, the structure may become stabilized by inter/intra glycan strand cross-links. The model also predicts that the cell wall is likely only one intact cable thick with partially hydrolyzed cables also present externally. Solidstate NMR data obtained for Staphylococcus aureus PG, which contains an interpeptide pentaglycyl bridge, show that the spatial arrangement of the polymer in staphylococci may be even more complex. Partial characterization of the structure was achieved by measuring spin diffusion from ${ }^{(13)} \mathrm{C}$ labels in pentaglycyl crosslinking segments to natural-abundance ${ }^{(13)} \mathrm{C}$ in the surrounding intact cell walls. The measurements were performed using a version of Centerband-Only Detection of EXchange (CODEX). The CODEX spin diffusion rates established that the pentaglycyl bridge of one peptidoglycan repeat unit of $S$. aureus is within 5 angstroms of the glycan chain of another repeat unit, which 
shows surprising proximity compared to earlier theoretical considerations and was interpreted in terms of a model for the peptidoglycan lattice in which all peptide stems in a plane perpendicular to the glycan main chain are parallel to one another (Sharif et al., 2009).

This minireview reflects the most recent achievements in research on peptidoglycan, with focus on modifications of the glycan chains and the spatial organization of the polymer.

\section{Literature}

Andre G., S. Kulakauskas, M.P. Chapot-Chartier, B. Navet, M. Deghorain, E. Bernard, P. Hols and Y.F. Dufrêne. 2010. Imaging the nanoscale organization of peptidoglycan in living Lactococcus lactis cells. Nat. Commun. 1: 1-8.

Araki Y., S. Fukuoka, S. Oba and E. Ito. 1971. Enzymatic deacetylation of $\mathrm{N}$-acetylglucosamine residues in peptidoglycan from Bacillus cereus cell walls. Biochem. Biophys. Res. Commun. 45: 751-758.

Atrih A. and S.J. Foster. 2001. In vivo roles of the germinationspecific lytic enzymes of Bacillus subtilis 168. Microbiology 147: 2925-2932.

Bera A., S. Herbert, A. Jakob, W. Vollmer and F. Gotz. 2005. Why are pathogenic staphylococci so lysozyme resistant? The peptidoglycan $O$-acetyltransferase OatA is the major determinant for lysozyme resistance of Staphylococcus aureus. Mol. Microbiol. 55: 778-787.

Bernard E., T. Rolain, P. Courtin, A. Guillot, P. Langella, P. Hols and M.P. Chapot-Chartier. 2011 Characterization of O-Acetylation of $\mathrm{N}$-Acetylglucosamine: A Novel structural variation of bacterial peptidoglycan. J. Biol. Chem. 286: 23950-23958.

Blackburn N.T. and A.J. Clarke. 2001. Identification of four families of peptidoglycan lytic transglycosylases. J. Mol. Evol. 52: 78-84. Beveridge T.J. 1995. The periplasm space and the periplasm in gram-positive and gram-negative bacteria. ASM News. 61: 125-130. Boneca I.G., O. Dussurget, D. Cabanes, M.A. Nahori, S. Sousa et al. 2007. A critical role for peptidoglycan $\mathrm{N}$-deacetylation in Listeria evasion from the host innate immune system. Proc. Natl. Acad. Sci. USA. 104: 997-1002.

Bugg T.D.H., D. Braddick, C.G. Dowson and D. I. Roper. 2011. Bacterial cell wall assembly: still an attractive antibacterial target. Trends Biotech. 29: 167-173.

Cava F., M.A. de Pedro, H. Lam, B.M. Davis and M.K. Waldor. 2011. Distinct pathways for modification of the bacterial cell wall by non-canonical D-amino acids. EMBO J. 30: 3442-3453.

Cloud-Hansen K.A., S.B. Peterson, E.V. Stabb, W.E. Goldman, M.J. McFall-Ngai and J. Handelsman. 2006. Breaching the great wall: peptidoglycan and microbial interactions. Nat. Rev. Microbiol. 9: 710-716.

Corr S.C. and L.A. O'Neill. 2009. Listeria monocytogenes infection in the face of innate immunity. Cell. Microbiol. 11: 703-709.

Coulombe F., M. Divangahi, F. Veyrier, L. de Léséleuc, J.L. Gleason et al. 2009. Increased NOD2-mediated recognition of N-glycolyl muramyl dipeptide. J. Exp. Med. 206: 1709-1716.

Crisostomo M.I., W. Vollmer, A.S. Kharat, S. Inhulsen, F. Gehre, S. Buckenmaier and A. Tomasz. 2006. Attenuation of penicillin resistance in a peptidoglycan $O$-acetyl transferase mutant of Streptococcus pneumoniae. Mol. Microbiol. 61: 1497-1509.

Cummins C.S. and H. Harris. 1956. The chemical composition of the cell wall in some gram-positive bacteria and its possible value as a taxonomic character. J. Gen. Microbiol. 14: 583-600.

Davis K. M. and J.N. Weiser. 2011 Modifications to the peptidoglycan backbone help bacteria to establish infection. Infect. Immun. 79: 562-570.
Dmitriev B.A., F.V. Toukach, K.J. Schaper, O. Holst, E.T. Rietschel and S. Ehlers. 2003. Tertiary structure of bacterial murein: the scaffold model. J. Bacteriol. 185: 3458-3468.

Dmitriev B.A., F.V. Toukach, O. Holst, E.T. Rietschel and S. Ehlers. 2004. Tertiary structure of Staphylococcus aureus cell wall murein. J. Bacteriol. 186: 7141-7148.

Dmitriev B.A., F. Toukach and S. Ehlers. 2005. Towards a comprehensive view of the bacterial cell wall. Trends Microbiol. 13: 569-754. Dowd M.M., B. Orsburn and D.L. Popham. 2008. Cortex peptidoglycan lytic activity in germinating Bacillus anthracis spores. J. Bacteriol. 190: 4541-4548.

Dziarski R. and D. Gupta. 2010. Review: Mammalian peptidoglycan recognition proteins (PGRPs) in innate immunity. Innate Immun. 16: 168-174.

Fittipaldi N., T. Sekizaki, D. Takamatsu, D.-P. M. de la Cruz, J. Harel, N.K. Bui, W. Vollmer and M. Gottschalk. 2008 Significant contribution of the pgdA gene to the virulence of Streptococcus suis. Mol. Microbiol. 70(5):1120-1135.

Gan L., S. Chen and G. L. Jensen. 2008. Molecular organization of Gram-negative peptidoglycan. Proc. Natl Acad. Sci. USA 105: 18953-18957.

Gilmore M.E., D. Bandyopadhyay, A.M. Dean, S.D. Linnstaedt and D.L. Popham. 2004. Production of muramic delta-lactam in Bacillus subtilis spore peptidoglycan. J. Bacteriol. 186: 80-89.

Glauner B. 1988 Separation and quantification of muropeptides with high-performance liquid chromatography. Anal. Biochem. 172: 451-464.

Graham L.L., T.J. Beveridge and N. Nanninga. 1991. Periplasmic space and the concept of periplasm. Trends. Biochem. Sci. 16: 328-329.

Gram H.C. 1884. Uber die isolierte Farbung der Schizomyceten in Schnitt- and Trockenpraparaten. Fortschritte der Medizin 2: 185-189. Hayhurst E. J., L. Kailas, J. K. Hobbs and S. J. Foster. 2008. Cell wall peptidoglycan architecture in Bacillus subtilis. Proc. Natl. Acad. Sci. USA 105: 14603-14608.

Heffron J.D., N. Sherry and D.L. Popham. 2011. In vitro studies of peptidoglycan binding and hydrolysis by the Bacillus anthracis germination-specific lytic enzyme SleB. J. Bacteriol. 193: 125-131. Heidrich C., A. Ursinus, J. Berger, H. Schwarz and J.V. Höltje. 2002. Effects of multiple deletions of murein hydrolases on viability, septum cleavage, and sensitivity to large toxic molecules in Escherichia coli. J. Bacteriol. 184: 6093-6099.

Höltje J.V., D. Mirelman, D. Sharon and U. Schwarz. 1975. Novel type of murein transglycosylase in Escherichia coli. J. Bacteriol. 124: 1067-1076.

Höltje J.V. 1998 Growth of the stress-bearing and shape-maintaining murein sacculus of Escherichia coli. Microbiol. Mol. Biol. Rev. 62: 181-203.

Höltje JV and Glauner B. 1990. Structure and metabolism of the murein sacculus. Res. Microbiol. 141: 75-89.

Kietzman C. and E. Tuomanen. 2011. PGRPs kill with an ancient weapon. Nat. Med. 17: 665-666.

Korsak D., W. Vollmer and Z. Markiewicz. 2005. Listeria monocytogenes EGD lacking penicillin-binding protein 5 (PBP5) produces a thicker cell wall. FEMS Microbiol. Lett. 251: 281-288.

Korsak D., Z. Markiewicz, G.O. Gutkind and J.A. Ayala. 2010. Identification of the full set of Listeria monocytogenes penicillinbinding proteins and characterization of PBPD2 (Lmo2812). BMC Microbiol. 10: 239.

Laaberki M.H., J. Pfeffer, A.J. Clarke and J. Dworkin. 2011. O-Acetylation of peptidoglycan is required for proper cell separation and S-layer anchoring in Bacillus anthracis. J. Biol. Chem. 286: 5278-5288.

Markiewicz Z., B. Glauner and U.Schwarz. 1983. Murein structure and lack of DD- and LD-carboxypeptidase activities in Caulobacter crescentus. J. Bacteriol. 156: 649-655. 
Markiewicz Z. 1993. The Structure and Functions of the Cell Envelope (in Polish). Polish Scientific Publishers PWN, Warsaw. Matias V.R. and T.J. Beveridge. 2005. Cryo-electron microscopy reveals native polymeric cell wall structure in Bacillus subtilis 168 and the existence of a periplasmic space. Mol. Microbiol. 56: $240-251$.

Matias V.R. and T.J. Beveridge. 2006. Native cell wall organization shown by cryo-electron microscopy confirms the existence of a periplasmic space in Staphylococcus aureus. J. Bacteriol. 188: 1011-1021. Matias V.R. and T.J. Beveridge. 2008. Lipoteichoic acid is a major component of the Bacillus subtilis periplasm. J. Bacteriol. 190: 7414-7418.

Merchante R., H.M. Pooley and D. Karamata. 1995. A periplasm in Bacillus subtilis. J. Bacteriol. 177: 6176-6183.

Meroueh S.O., K.Z. Bencze, D. Hesek, M. Lee, J.F. Fisher, T.L. Stemmler and S. Mobashery. 2006 Three-dimensional structure of the bacterial cell wall peptidoglycan. Proc. Natl. Acad. Sci. USA 103: 4404-4409.

Meyrand M., A. Boughammoura, P. Courtin, C. Mezange, A. Guillot and M-P. Chapot-Chartier. 2007. Peptidoglycan $\mathrm{N}$-acetylglucosamine deacetylation decreases autolysis in Lactococcus lactis. Microbiology 153: 3275-3285.

Moynihan P.J. and A.J. Clarke. 2010. O-acetylation of peptidoglycan in gram-negative bacteria: identification and characterization of peptidoglycan O-acetyltransferase in Neisseria gonorrhoeae. J. Biol. Chem. 285: 13264-13273.

Park, J.T. and M.J. Johnson. 1949. Accumulation of labile phosphorus in Staphylococcus aureus grown in the presence of penicillin. J. Biol. Chem. 179:585-592.

Pavelka M.S. Jr. 2007. Another brick in the wall. Trends Microbiol. 15: 147-149.

Peltier J., P. Courtin, I. El Meouche, L. Lemee, M.P. Chapot-Chartier and J.L. Pons. 2011. Clostridium difficile has an original peptidoglycan structure with high level of $\mathrm{N}$-acetylglucosamine deacetylation and mainly 3-3 cross-links. J. Biol. Chem. 286: 20953-20962.

Pink D., J. Moeller, B. Quinn, M. Jericho and T. Beveridge. 2000. On the architecture of the gram-negative bacterial murein sacculus. J. Bacteriol. 182: 5925-5930.

Popham D.L., J. Helin, C.E. Costello and P. Setlow. 1996. Analysis of the peptidoglycan structure of Bacillus subtilis endospores. J. Bacteriol. 178: 6451-6458.

Popowska M., M. Kusio, P. Szymanska and Z. Markiewicz. 2009. Inactivation of the wall-associated de- $N$-acetylase (PgdA) of Listeria monocytogenes results in greater susceptibility of the cells to induced autolysis. J. Microbiol. Biotechnol. 9: 932-945.

Popowska M., M. Osinska and M. Rzeczkowska. 2011. N-acetylglucosamine-6-phosphate deacetylase (NagA) of Listeria monocytogenes EGD, an essential enzyme for the metabolism and recycling of amino sugars. Arch. Microbiol. Accepted Aug. 27 DOI: 10.1007/ s00 203-011-0752-3.

Rae C.S., A. Geissler, P.C. Adamson and D.A. Portnoy. 2011. Mutations of the Listeria monocytogenes peptidoglycan $\mathrm{N}$-deacetylase and $O$-acetylase result in enhanced lysozyme sensitivity, bacteriolysis and hyper-induction of innate immune pathways. Infect. Immun. 79: 3596-3608.

Raymond J.B., S. Mahapatra, D.C. and M.S. Jr. Pavelka. 2005. Identification of the namH gene, encoding the hydroxylase responsible for the $\mathrm{N}$-glycolylation of the mycobacterial peptidoglycan. J. Biol. Chem. 280: 326-333.

Reith J. and C. Mayer C. 2011. Peptidoglycan turnover and recycling in Gram-positive bacteria Appl. Microbiol. Biotechnol. Jul 28. [Epub ahead of print]

Rogers H.J. 1974. Peptidoglycans (mucopeptides): structure, function, and variations. Ann. N. Y. Acad. Sci. 35: 29-51.

Salton M. R.J. 1952. Cell Wall of Micrococcus Lysodeikticus as the substrate of lysozyme. Nature 170: 746-748.
Salton M.R. and R.W. Horne. 1951 Studies of the bacterial cell wall. II. Methods of preparation and some properties of cell walls. Biochim. Biophys. Acta 7: 177-197.

Salton M.R. 1957. Cell-wall amino-acids and amino-sugars. Nature 180: 338-339.

Schäberle T.F., W. Vollmer, H.J. Frasch, S. Hüttel, A. Kulik, M. Röttgen, A.K. von Thaler, W. Wohlleben and E. Stegmann. 2011. Self-resistance and cell wall composition in the glycopeptide producer Amycolatopsis balhimycina. Antimicrob. Agents Chemother. 55: 4283-4289.

Scheurwater E.M. and Clarke A.J. 2008. The C-terminal domain of Escherichia coli YfhD functions as a lytic transglycosylase. J. Biol. Chem. 283: 8363-8373.

Scheurwater E.M. and L.L Burrows. 2011. Maintaining network security: how macromolecular structures cross the peptidoglycan layer. FEMS Microbiol. Lett. 318: 1-9.

Schleifer K.H. and O. Kandler. 1972. Peptidoglycan types of bacterial cell walls and their taxonomic implications. Bacteriol. Rev. 36: 407-477.

Shaik M.M., L. Cendron, R. Percudani and G. Zanotti. 2011. The structure of Helicobacter pylori HP0310 reveals an atypical peptidoglycan deacetylase. PLoS One. 6: e19207.

Sharif S., M. Singh, S.J. Kim and J. Schaefer. 2009. Staphylococcus aureus peptidoglycan tertiary structure from carbon-13 spin diffusion. J. Am. Chem. Soc. 131(20):7023-7030.

Sharif S, Singh M., Kim S. J. and J Schaefer. 2009. Staphylococcus aureus peptidoglycan tertiary structure from carbon-13 spin diffusion. J. Am.Chem. Soc. 131: 7023-7030.

Verwer R.W., N. Nanninga, W. Keck and U. Schwarz. 1978. Arrangement of glycan chains in the sacculus of Escherichia coli. J. Bacteriol. 136:723-729.

Vollmer W., D. Blanot and M.A. de Pedro. 2008. Peptidoglycan structure and architecture. FEMS Microbiol. Rev. 322: 149-167.

Vollmer W. and A. Tomasz. 2002. Peptidoglycan $N$-acetylglucosamine deacetylase, a putative virulence factor in Streptococcus pneumoniae. Infect. Immun. 70: 7176-7178.

Vollmer W. and J.V. Höltje. 2004. The architecture of the murein (peptidoglycan) in gram-negative bacteria: vertical scaffold or horizontal layer(s)? J. Bacteriol. 186: 5978-5987.

Vollmer W. and S.K.J. Seligman. 2010 Architecture of peptidoglycan: more data and more models. Trends Microbiol. 18: 59-66.

Watterlot L., M. Meyrand, N. Gaide, P. Kharrat, S. Blugeon, J.J. Gratadoux, M.J. Flores, P. Langella, M.P. Chapot-Chartier and L.G. Bermúdez-Humarán. 2010. Variations of $N$-acetylation level of peptidoglycan do not influence persistence of Lactococcus lactis in the gastrointestinal tract. Int. J. Food Microbiol. 144: 29-34. Weadge J.T., J.M. Pfeffer and A.J. Clarke. 2005. Identification of a new family of enzymes with potential $N$-acetylpeptidoglycan esterase activity in both Gram-positive and Gram-negative bacteria. BMC Microbiol. 5: 49.

Work E. 1957. Biochemistry of the bacterial cell wall. Nature 179: 841-847.

Work E. 1961. The mucopeptides of bacterial cell walls. A review. J. Gen. Microbiol. 25:169-189.

Work E. 1969. Biochemistry of bacterial cell walls. Lab. Pract. 18: 831-838.

Yoon J, Y. Matsuo, S. Matsuda, H. Kasai and A. Yokota. 2010. Cerasicoccus maritimus sp. nov. and Cerasicoccus frondis sp. nov., two peptidoglycan-less marine verrucomicrobial species, and description of Verrucomicrobia phyl. nov., nom. rev. J. Gen. Appl. Microbiol. 56: 213-222.

Zuber B., M. Haenni, T. Ribeiro, K. Minnig, F. Lopes, P. Moreillon and J. Dubochet. 2006. Granular layer in the periplasmic space of gram-positive bacteria and fine structures of Enterococcus gallinarum and Streptococcus gordonii septa revealed by cryo-electron microscopy of vitreous sections. J. Bacteriol. 188: 6652-6660. 